\title{
Evolution of Genuine Cross-Correlation Strength of Focal Onset Seizures
}

\author{
Markus F. Müller, *† Gerold Baier,ł Yurytzy López Jiménez, * Arlex O. Marín García, * \\ Christian Rummel, $\S$ and Kaspar Schindler
}

\begin{abstract}
Summary: To quantify the evolution of genuine zero-lag cross-correlations of focal onset seizures, we apply a recently introduced multivariate measure to broad band and to narrow-band EEG data. For frequency components below $12.5 \mathrm{~Hz}$, the strength of genuine cross-correlations decreases significantly during the seizure and the immediate postseizure period, while higher frequency bands show a tendency of elevated cross-correlations during the same period. We conclude that in terms of genuine zero-lag cross-correlations, the electrical brain activity as assessed by scalp electrodes shows a significant spatial fragmentation, which might promote seizure offset.
\end{abstract}

Key Words: focal onset seizures, linear correlation analysis, genuine and random correlations, multivariate analysis.

( Clin Neurophysiol 2011;0: 1-13)

$F_{b}$ r the quantitative analysis of complex systems like the human brain, development and application of sophisticated tools of time series analysis are indispensable. During the past decade, especially the application of nonlinear measures became popular, mostly, because it was supposed that the mechanisms underlying an EEG signal are nonlinear or even chaotic (Babloyantz and Destexhe, 1986; Babloyantz et al., 1985; Lehnertz et al., 2000; Nan and Jinghua, 1988; Rapp et al., 1985; Skarda and Freeman, 1987). Hence, a multitude of different techniques like the estimation of the largest Lyapunov exponent (Iasemidis et al., 2003), the correlation dimension (Lehnertz and Elger, 1998), generalized correlations via mutual information (Mars and Lopes da Silva, 1983), nonlinear regression (Pijn and Lopes da Silva, 1993; Wendling et al., 2001), or synchronization measures (Arnhold et al., 1999; Bhattacharya, 2001; Mormann et al., 2000; Rosenblum et al., 2004; Stam and van Dijk, 2002) have been applied. A comprehensive review of the most prominent techniques applied to EEG analysis can be found in the review by Stam (2005). All the before-mentioned methods are univariate or bivariate, evaluating properties of single or the interplay of pairs of data channels of a multivariate EEG recording. However, if

From the *Facultad de Ciencias, Universidad Autónoma del Estado de Morelos, Cuernavaca, Mexico; †Centro Internacional de Ciencias, Universidad Nacional Autónoma de México, Cuernavaca, Morelos, Mexico; \$Manchester Interdisciplinary Biocentre, University of Manchester, Manchester, United Kingdom; and $\S$ Department of Neurology, Inselspital Bern, University Hospital, University of Bern, Bern, Switzerland.

This work was supported by CONACyT, Mexico (Proj. No. 48500) and by Deutsche Forschungsgemeinschaft, Germany (grant RU1401/1-2, RU1401/2-1) and by the Swiss National Science Foundation (SNF No. 320030_122010). G.B. is supported by EPSRC/BBSRC.

Address correspondence and reprint requests to Markus F. Müller, PhD, Facultad de Ciencias, UAEM, Av. Universidad 1001, 62209, Cuernavaca, Morelos, Mexico; e-mail: muellerm@buzon.uaem.mx.

Copyright (C) 2011 by the American Clinical Neurophysiology Society

ISSN: 0736-0258/11/0000-0001 one aims at detecting relationships between $\mathrm{m}>2$ data channels and at quantifying the total strength of interrelations, a multivariate approach seems most promising. This conjecture is motivated by the typical network structure of mammalian brains (Bartolomei et al., 2001; Netoff et al., 2004; Ponten et al., 2007; Sporns and Zwi, 2004), where interconnections lead to more complex structures than those given by a set of bivariate relations.

A linear multivariate approach was applied in the study by Schindler et al. (2007b) to intracranial recordings of 100 focal onset seizures of 60 patients to study the evolution of zero-lag cross-correlations between the signals measured by different electrodes. The authors provided evidence that the total amount of cross-correlation remains approximately unchanged throughout the first part of the seizures but increases gradually during the final part. Also, based on the earlier work by Topolnik et al. (2003), the authors proposed that increasing neuronal synchronization during epileptic seizures might be an emergent self-regulatory mechanism for seizure offset. As one possible molecular mechanism that could promote seizure offset, it was proposed that $\mathrm{Na}^{+}$- and $\mathrm{Ca}^{2+}$-activated potassium currents overcome hyperpolarization-activated depolarizing ionic currents (Topolnik et al., 2003). It was speculated that activation of potassium currents might be most effective in hyperpolarizing neuronal membranes, thereby suppressing ictal activity when occurring simultaneously in spatially extended neuronal networks (Schindler et al., 2007b). Thus, it was postulated that the increase of correlated EEG activity before seizure offset could be an active seizure offset mechanism and not simply an epiphenomenon. Further evidence for this hypothesis was put forward in Schindler et al. (2007a), where the evolutions of six status epilepticus EEG recordings were studied.

The studies presented in Schindler et al. $(2007 a, 2007 b)$ are based on the eigenvalue spectrum of the zero-lag cross-correlation matrix with Pearson correlation coefficients as elements. Naturally, all matrix elements are estimated over a finite time window, usually chosen as small as possible to obtain an optimal time resolution. However, any measure, whose rigorous mathematical definition contains integrals over an infinite range bears deficiencies, when applied to data segments of finite lengths. This is particularly true for the Pearson coefficient, which is generally contaminated by random correlations (e.g., Müller et al., 2005, 2006; Plerou et al., 2002).

Importantly, the amount of random correlations may change, when the frequency contents of the signals, that is, the power spectra vary (Müller et al., 2008a; Rummel et al., 2010). This effect is illustrated in Fig. 1A, where the distribution of nondiagonal elements of the correlation matrix is displayed for surrogate data of different segments of a 19-channel scalp EEG recording. The surrogates conserve the power spectra of the original data, while all linear as well as nonlinear relationships between the data channels are destroyed (see Surrogate Data and Estimating the Strength of Genuine Cross- 
Correlation). The EEG segments chosen for this illustration are from a temporal lobe seizure in case of the solid line histogram and from the immediate postseizure period in case of the dashed probability distribution. The power spectra (of electrode Fz) of these epochs are shown in Fig. 1B. In case of immediate postseizure period (dashed line histogram), the amount of slow frequencies is considerably higher than during the seizure, while it contains less contribution from higher frequencies.

As expected for uncorrelated data, both distributions shown in Fig. 1A are bell shaped and centered around zero. However, the width of the distribution drawn from the data with a higher contribution of slow frequencies is much larger, providing a higher probability for the (incidental) appearance of large cross-correlation coefficients. However, the widths of the 2 distributions shown in Fig. 1A do not represent any genuine cross-correlations of the data. They are unique because a large value for the correlation coefficient may appear by chance, if estimated from a finite amount of data points. Therefore, this phenomenon is usually referred to as "random correlations" (Plerou et al., 2002).

One of the central concerns of the present article is to discriminate random from genuine components of cross-correlation (avoiding the influence of the spectral content of the signals) and to
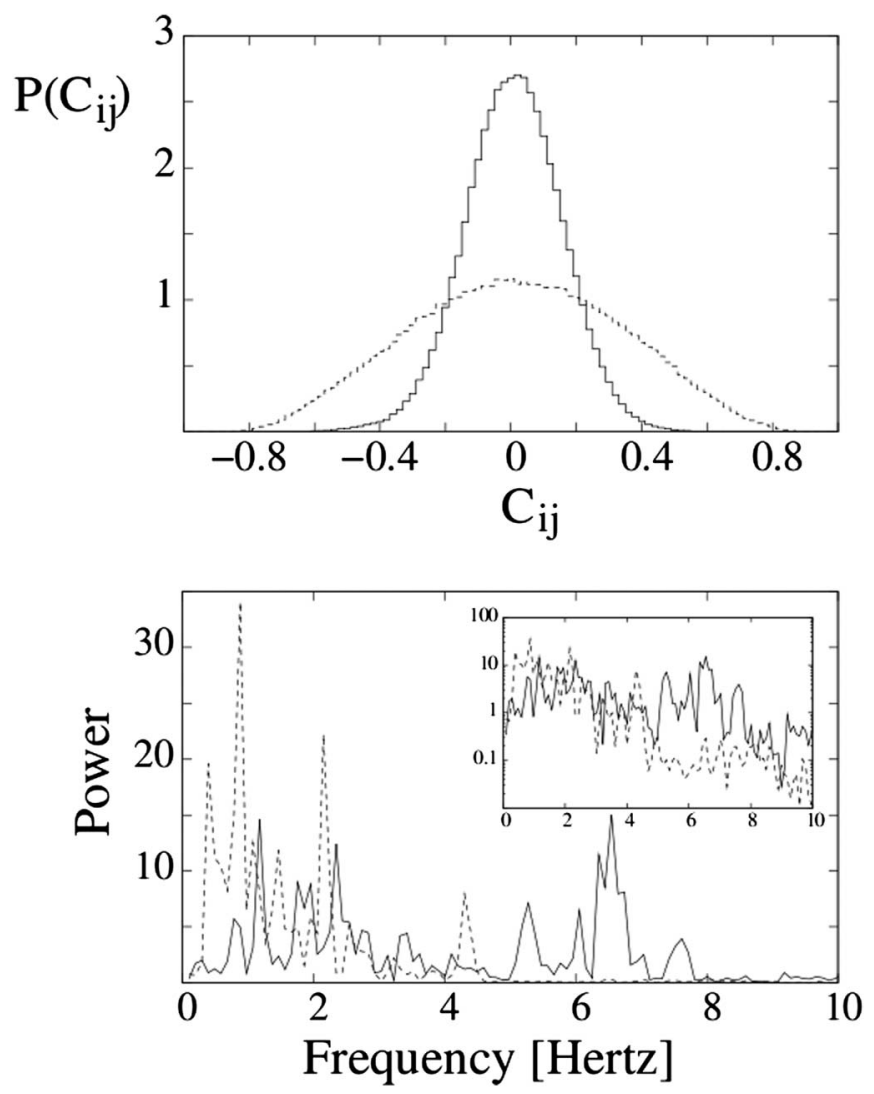

FIG. 1. A, Distribution of the nondiagonal elements of the correlation matrix constructed from surrogate data generated from selected segments of the EEG recording of seizure 2. Solid line histogram indicates seizure period, and dashed line indicates immediate postseizure epoch. B, Power spectra from selected segments of seizure 2: Solid line reflects the seizure period, and dashed line reflects the postseizure epoch of electrode Fz. The inlet shows the same data on a logarithmic scale. probe the hypothesis formulated in (Schindler et al., 2007a, 2007b), where an increase of zero-lag correlations was considered as an active self-regulatory mechanism for seizure offset. For this purpose, we focus on a recently introduced measure that quantifies the strength of genuine zero-lag cross-correlations (CCS) (Müller et al., 2008a). This approach is applied to 12 scalp EEG recordings of 5 patients containing focal onset seizures to characterize the evolution of the genuine cross-correlation strength (CCS) from the preseizure to the postseizure period. We found that genuine and random correlations behave very differently, an effect that, to the best of our knowledge, has not been accounted for in previous approaches. Furthermore, we test whether the results change, when different spatial scales, as those covered by scalp or foramen ovale electrodes, are assessed. As different frequency contributions may show distinct behavior on different spatial scales, we repeat the analysis for band pass-filtered data.

\section{METHODS}

We first discuss why we focus in the present work on a linear approach and summarize the definition and some important mathematical properties of the zero-lag cross-correlation matrix. Then, we describe the surrogate data used in this article, and the way they are invoked (1) to test for the null hypothesis of uncorrelated data and (2) to define a (normalized) measure that quantifies the strength of genuine cross-correlations. Thereafter, we describe the statistical tests to evaluate changes from the pre- to the postseizure period. Finally, we report relevant details about data acquisition and preprocessing and discuss briefly the influence of the EEG reference on the spectrum of eigenvalues of the correlation matrix.

\section{Selection of an Appropriate Bivariate Measure}

The multivariate method used in this article is based on a bivariate interrelation measure. Naturally, its performance and the interpretation of the results depend crucially on the choice of this measure. Therefore, we include a brief discussion of this issue.

In principle, one expects that the dynamics of a complex system consisting of a huge number of coupled excitable oscillators is essentially nonlinear and probably chaotic. As for the mathematical description of neurons, nonlinear differential equations are used (see e.g., (Keener and Sneyd, 1998)), and this seems particularly true for the human brain. Consequently, one expects that measures sensitive to nonlinear features in experimental data perform better than those that extract only linear relationships.

However, as the number of degrees of freedom is of the order of $10^{10}$, the underlying attractor is expected to be high dimensional and, in addition, experimental signals are generally noise contaminated. Due to the non-stationarity of the EEG only comparably small data segments can be used for estimating the characteristic quantities. Thus it seems impossible to dissolve such high dimensional deterministic structures from experimental recordings. Besides in general, it is a delicate issue to apply sophisticated measures requiring the adjustment of several technical parameters (e.g., as any measure based on a phase space reconstruction via embedding) in an unsupervised manner using a running window approach (Kantz and Schreiber, 1997). These might be the main reasons for the results presented in various studies (Casdagli, 1992; Palus, 1996; Pijn et al., 1991, 1997; Stepien, 2002; Theiler, 1995; Theiler and Rapp, 1996), where it has been reported that EEG recordings are well described by linear stochastic processes and little or no evidence for the presence of nonlinear or even chaotic determinism could be found. 
Furthermore, in a quantitative comparison of different linear and nonlinear interrelation measures using data derived from well defined test frameworks, it turns out that linear measures perform equally good or even better than nonlinear methods in detecting the coupling between nonlinear or even chaotic units (Ansari-Asl et al., 2006; Kreuz et al., 2007). Although it results that there is no universal method that outperforms others in all studied cases, it was shown that in the context of seizure prediction and detection linear approaches are highly competitive (Jerger et al., 2002; Mormann et al., 2005). The authors of Ansari-Asl et al. (2006) explicitly recommend to apply correlation measures as a first attempt to characterize the functional coupling in systems under study.

Finally, we remark that several popular measures may lead to erroneous results for the particular application of EEG analysis. As an example, we mention synchronization measures based on the Hilbert transformation. To yield interpretable results, the power spectrum of the signals should be narrow with only one prominent peak (Chavez et al., 2006; Pikovsky et al., 2001). Otherwise, the interpretation of the results obtained by synchronization measures may remain ambiguous and the term "synchronization" may lose its meaning (Chavez et al., 2006). Hence, a possible way to solve this problem would be the study of band pass-filtered data. However, band pass filtering may lead to a spurious increase of the degree of phase synchronization (Xu et al., 2006). For these reasons, we concentrate on the study of linear cross-correlations estimated by the Pearson coefficient.

\section{The Zero-Lag Correlation Matrix}

For a measured multivariate time series $\mathrm{X}_{i}(t)(i=1 \ldots M)$, the zero-lag correlation matrix $\hat{C}$ (e.g., (Anderson, 2003; Morrison, 2005)) is constructed by first normalizing each data channel within a given window of length $\mathrm{T}$ data points to zero mean and unit variance

$$
\tilde{X}_{i}(t)=\frac{X_{i}(t)-\left\langle X_{i}\right\rangle_{t}}{\sigma_{i}^{t}}
$$

and then evaluating the matrix elements as

$$
\tilde{C}_{i j}(t)=\frac{1}{T} \sum_{t=1}^{T} \tilde{X}_{i}(t) \tilde{X}_{j}(t)=\left\langle\tilde{X}_{i} \tilde{X}_{j}\right\rangle_{t}
$$

In the last 2 equations, averages are denoted by \langle\rangle and the standard deviations by $\sigma$. They are calculated over the time window $T$. The $M$ eigenvalues $\lambda_{i}$ and eigenvectors $\vec{v}_{i}$ of $\hat{C}$ are obtained by solving the equation

$$
\hat{C} \vec{v}_{i}=\lambda_{i} \vec{v}_{i}
$$

The index of the eigenvalues indicates an increasing order $\lambda_{i} \leq \lambda_{i+1}$. With the help of the eigenvalues and eigenvectors, it is possible not only to separate random from nonrandom contributions but also to capture interrelations between $2 \leq \mathrm{m} \leq \mathrm{M}$ signals (Conlon et al., 2009a, 2009b; Müller et al., 2005, 2006; Rummel et al., 2007a; Rummel, 2008).

In general, an increase of (random as well as genuine) crosscorrelations leads to a broadening of the eigenvalue spectrum, while for less correlated data, the eigenvalues are closely distributed around unity. This broadening is caused by repulsions between eigenvalues, which might occur at any location along the whole spectrum. It turns out that the repulsion pattern between the $\lambda_{i}$ is a characteristic feature for any particular correlation structure (Müller et al., 2005). An illustrative explanation on how the eigenvalues react under different conditions can also be found in Müller et al. (2008b) and Schindler et al. (2007a, 2007b). If one aims to distinguish between random and genuine correlations, tools from random matrix theory can be used (Baier et al., 2007; Müller et al., 2006), to detect those parts of the eigenvalue spectrum where the $\lambda_{i}$ are altered by nonrandom repulsions. Unfortunately, to apply these techniques, the generation of an ensemble of correlation matrices over a stationary data segment is needed, where the significance of the results depends crucially on the size of this ensemble. Especially for highly nonstationary data sets, such as EEG recordings, this requirement can hardly be fulfilled. Alternatively, surrogate data can be used to test the null hypothesis of linearly uncorrelated dynamics conserving the property that the derived measure is sensitive to local properties of the eigenvalue spectrum. This strategy is followed in the present article.

\section{Surrogate Data and Estimating the Strength of Genuine Cross-Correlation}

Surrogate data are widely used for testing whether empirical data contain any nonlinear deterministic structure (Kantz and Schreiber, 1997). In the present case, we use surrogate data to test for the null hypothesis that the EEG does not contain any linear, zero-lag cross-correlations. As the amount of random correlations depends on the frequency content of a signal, we use iterative amplitude adjusted Fourier transform surrogates (Schreiber and Schmitz, 2000), which conserve the power spectrum and the amplitude distribution individually for each of the $\mathrm{M}$ data channels but destroy all linear and nonlinear relationships between the M signals. All surrogate data used in this publication were generated by the freely available TISEAN software package (Hegger et al., 1999). Additionally, we checked the performance of shift surrogates as proposed in Netoff and Schiff (2002), obtaining quantitatively equivalent results.

While the distribution of eigenvalues computed from the surrogates reflects random cross-correlations, the eigenvalue spectra obtained from the original data represent both random and genuine contributions. Hence, any significant deviation of the eigenvalues calculated from the original data $\lambda_{i}$ from those obtained from the surrogates $\lambda_{s}$ indicates genuine cross-correlations. As distortions of the eigenvalue spectrum caused by genuine cross-correlations may occur locally at any site of the eigenvalue spectrum, one has to test for significant differences for each of the eigenvalues independently. This is important because any global measure derived from comparing the distribution of all eigenvalues or any kind of spectral average may wipe out the effects induced by genuine cross-correlations and, hence, may strongly reduce the sensitivity of the derived measure. The last statement is illustrated in Müller et al. (2006), where the nearest neighbor spacing distribution was used as a test statistics.

To evaluate whether a deviation is significant or simply caused by statistical fluctuations, we apply a two-sided $U$-rank test of Mann-Whitney-Wilcoxon (Mann and Whitney, 1947; Wilcoxon, 1945), which checks whether two samples stem from the same population. As the Mann-Whitney-Wilcoxon $U$-test is nonparametric, no implicit assumption about the probability distribution of the eigenvalues is made. Additionally, it can be used for comparably small samples, which reduces the numerical effort for producing a large number of surrogates. A direct measure for the genuine 
CCS is then given by the sum of all significant eigenvalue deviations along the whole spectrum:

$$
\mathrm{CCS}=\frac{\sum_{i=1}^{M}\left|\tilde{\lambda}_{i}-\tilde{\lambda}_{i}^{s}\right| s_{i}}{\sum_{i=1}^{M-1} \tilde{\lambda}_{i}^{s}+\left(M-\tilde{\lambda}_{M}^{s}\right)}
$$

where $\widetilde{\lambda}_{i}$ and $\widetilde{\lambda}_{i}^{s}$ denote the median of the set of the $i$-th eigenvalue calculated over a segment taken from the original data and the surrogates, respectively. The factor $s_{i}$ ensures that only significant deviations between the median values are taken into account. It is zero if the null hypothesis of statistical equivalence between $\lambda_{i}$ and $\widetilde{\lambda}_{i}^{s}$ cannot be rejected on a $1 \%$ significance level according to the MannWhitney-Wilcoxon $U$-test and is equal to one otherwise. For this statistical evaluation a Bonferroni correction has been applied.

The denominator in Eq. 4 properly normalizes the CCScoefficient such that it varies between zero and one, which can be easily seen by considering two limiting cases. If no genuine crosscorrelations are present, the eigenvalues calculated from the original data are statistically equivalent to those calculated from the surrogates and the CCS is equal to zero. However, if the system is maximally correlated (all time series are identical), the largest eigenvalue $\lambda_{M}=M$, while all others are equal to zero. In that case, numerator and denominator of Eq. 4 are identical and in consequence CCS $=1$. The definition Eq. 4 takes into account deviations along the whole spectrum of eigenvalues and is therefore also sensitive to detecting subtle correlations between only few data channels, which predominantly manifested a distortions at the lower edge of the eigenvalue spectrum (Müller et al., 2005, 2006).

The application of Eq. 4 requires an (possibly small) ensemble of correlation matrices derived from the original data (as well as from the surrogates). For this purpose, a window of length $\mathrm{T}$ is shifted with a certain step width over predefined data segments, as described in the section Statistical Evaluation of the Results. Then an ensemble is obtained by creating the zero-lag cross-correlation matrix (Eq. 2) for each of these windows.

\section{Estimation of Power Spectra}

To illustrate that changes of the power spectra of the EEG may cause dramatic changes in the amount of random correlations, we estimate occasionally the power spectra of the experimental recordings for comparison with time evolution of the eigenvalues. To this end, we apply a fast Fourier transform (Press et al., 2007) calculated over a window of 512 sampling points. This window was shifted with a step size of 50 data points over a given data segment as defined in Statistical Evaluation of the Results. Then the power, averaged over a predefined frequency band and over the segment, is calculated.

\section{Statistical Evaluation of the Results}

From the 12 EEG recordings, we selected sections of 1,500 seconds from long-term recordings, such that all contain an epileptic seizure starting approximately second 600 . Then segments of 2,000 data points (equivalent to 10 seconds) were defined and shifted with a step size of 1,000 sampling points along the recording. For each segment, 10 Fourier-based surrogates were computed. Then, we simultaneously analyzed the segments of the original data and those of the surrogates. For this purpose, we constructed the zero-lag correlation matrix over 200 data points (corresponding to 1 second), which was then shifted with a step width of 200 data points over the segment. In this way, we obtained an ensemble of 10 correlation matrices for the original data and another ensemble of 100 matrices for the surrogates. These matrices were diagonalized and the median of the sets of each eigenvalue $\widetilde{\lambda}_{i}$ and $\tilde{\lambda}_{i}^{s}$ were obtained for each segment. Then, the amount of genuine CCS within a data segment was calculated, where for each term of the sum in the numerator of Eq. 4, the Mann-Whitney-Wilcoxon $U$-test (Mann and Whitney, 1947; Wilcoxon, 1945) was performed to determine $s_{i}$.

Eventually, we evaluated relative changes of the genuine correlation strength along the time course from a preseizure to a postseizure period. To this end, we considered four intervals. The preseizure interval (PreS) contains the first 200 seconds of each recording, that is, it terminates at approximately 400 seconds before the seizure onset. The limits of the second interval $(S)$ are given by seizure onset and offset. Interval 3 contains 100 seconds starting at seizure offset (P1), and the period between seconds 1,300 and 1,500 defines interval $4(\mathrm{P} 2)$. Then, we tested whether the distributions of CCS coefficients obtained for all 12 seizures of different intervals were centered around the same value or if a significant tendency for a relative change between intervals could be detected. After normalizing the CCS coefficients of each seizure to the average value calculated over the four segments, we applied first a Friedman test. Afterward, we applied the two-sided median test (Siegel, 1988), which has the advantages to be nonparametric and that extreme events do not dominate the results.

\section{Patients, EEG Data Acquisition, and Preprocessing}

The EEG data were recorded from 5 patients (age range, 22-45 years), who had pharmacoresistant partial epilepsy with temporal lobe seizures and taking part in the program for presurgical evaluation of the Department of Neurology of the Inselspital of the University of Bern. This retrospective study was approved by the Ethics Committee of the Canton of Bern. Furthermore, all the patients gave written informed consent that the EEG data might be used for research and teaching purposes. Further information about the patients is displayed in Table 1. For scalp EEGs, standard 10 to 20 montage positions (American EEG Society, 1986) were used. In addition to scalp EEG, two foramen ovale electrodes were applied

TABLE 1. Information About Patients and Seizures

\begin{tabular}{lllcrl}
\hline Patient & Age & Sex & Seizure & $\begin{array}{c}\text { Duration } \\
\text { in Seconds }\end{array}$ & Comments \\
\hline 1 & 22 & M & 1 & 96 & Hippocampal sclerosis left \\
& & & 2 & 122 & \\
& & & 3 & 84 & \\
2 & 28 & F & 4 & 132 & Discrete hippocampal \\
& & & 5 & 204 & atrophy right \\
3 & 45 & F & 6 & 113 & Left hippocampal and \\
& & & 7 & 52 & amygdaloid structures \\
& & & & & removed \\
4 & 23 & M & 8 & 150 & Mesiotemporal sclerosis left \\
& & & 9 & 120 & \\
& & & 10 & 203 & \\
5 & 33 & M & 11 & 56 & Hippocampal sclerosis left \\
& & & 12 & 106 & \\
\hline
\end{tabular}


to record from mesiotemporal structures (Siegfried et al., 1985) because for these patients, scalp EEG alone was not sufficient to localize the site of seizure onsets. Foramen ovale electrodes of manufacturing type CAD-FO-B4 (Ad-Tech Medical Instrument, Corp, Racine, WI) were used. Each of these foramen ovale electrodes (Fo) had four contacts. The smaller the number of the Fo contact, the closer to the tip of the electrode it was positioned. Even contact numbers denote contacts of the right foramen ovale electrode, and odd numbers those of the left electrode. An EEG-1032 amplifier system was used. After passing an antialiasing filter with a cutoff frequency of $70 \mathrm{~Hz}$ and an attenuation of $24 \mathrm{~dB}$ /octave, the EEG signals were sampled at $200 \mathrm{~Hz}$ analog/digital conversion had a resolution of 16 bit. EEG seizure onsets and seizure offsets were visually assessed by an experienced electroencephalographer (K.S.) in bipolar montage. Fig. 2A shows the EEG signal of the 19 scalp and the 8 foramen ovale recordings of seizure 2. Seizure onset and offset are marked in the amplification of 15-second segments in Figs. 2B and 2C.
Only seizures with minimal artifacts (as judged by visual inspection) were included in the present study. The raw data were measured with respect to earlobe references. Because of the strong contamination of electrodes Fp1 and Fp2 with eye movements in some of the recordings, those contacts were excluded from the analysis in general. Instead, electrodes F9 and F10 were included. To avoid a dominant influence of large amplitudes in the "Fo"-channels, we normalized the data channels separately to zero mean and unit variance. To this end, we used a data window containing 1,000 data points, which was shifted with a maximal overlap over the recordings. Then, we transformed the EEG recording to global average montage because with the exception of the smallest eigenvalue, this specific montage turns out to distort the eigenvalue spectrum least (Rummel et al., 2007b).

To diminish the influence of muscle artifacts, we applied a low pass filter to the data with a cutoff frequency of $20 \mathrm{~Hz}$. Also a high pass filter with a cut off frequency of $0.5 \mathrm{~Hz}$ has been applied.

A

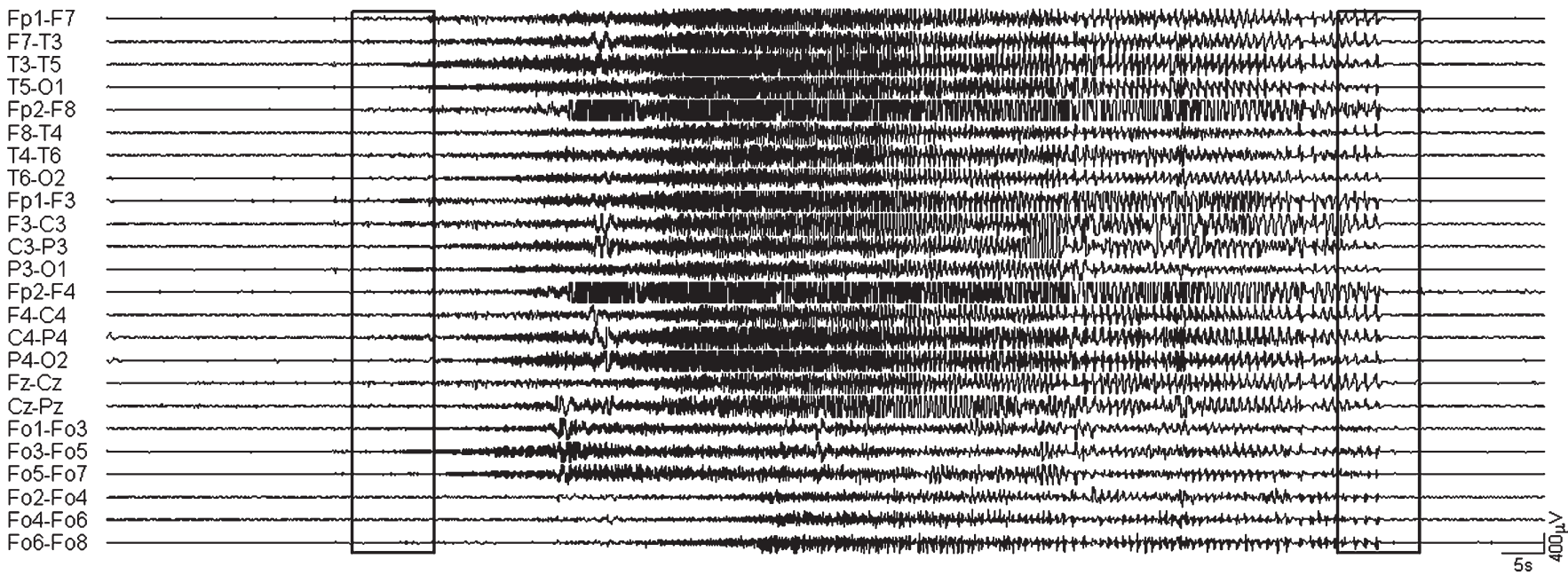

B

Fp1-F7
F7-T3
T3-T5
T5-01
Fp2-F8
F8-T4
T4-T6
T6-O2
Fp1-F3
F3-C3
C3-P3
P3-O1
Fp2-F4
F4-C4
C4-P4
P4-O2
Fz-Cz
Cz-Pz
F01-Fo3
F03-Fo5
F05-Fo7
Fo2-Fo4
Fo4-Fo6
Fo6-Fo8
C
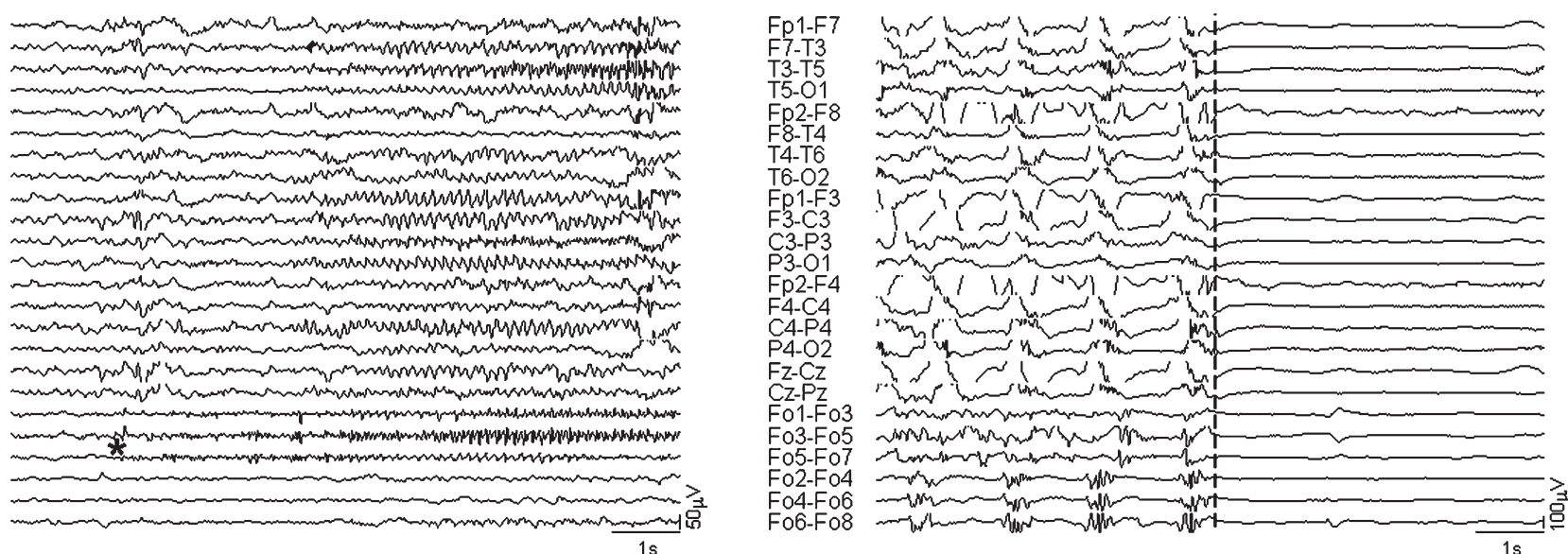

FIG. 2. EEG from seizure 2 of patient 1. A, Complete time course of the seizure. B, Seizure onset. C, Seizure end, as determined by visual inspection. Seizure onset is marked by a star symbol in B, and the foramen ovale electrode that show the first ictal EEG changes and seizure offset are marked by a vertical line in $\mathbf{C}$. 
These data sets are called "broad band signals" in the sequel.Eventually, band pass-filtered data were used, where the definition of the frequency bands was chosen according to Zschocke (2002): $\delta=(0.5,3.5) \mathrm{Hz}, \theta=(3.5,7.5) \mathrm{Hz}, \alpha=(7.5,12.5) \mathrm{Hz}, \beta=$ $(12.5,20) \mathrm{Hz}$. For this purpose a fourth order, Butterworth filter was applied in forward and backward direction to avoid a possible shifting of the signal phases. Furthermore, we checked the quality of the filtering process by comparing the original recording with the superposition of the filtered signals.

\section{Influence of the EEG Reference}

For the case of EEG analysis, an additional problem supervenes. EEG references may induce strong correlations within the data set that do not have any relation to correlations produced by the dynamics of the system. Even for otherwise uncorrelated data, in general, this leads to a complicated repulsion pattern of the eigenvalues, which may lead to an erroneous interpretation of the results. Furthermore, these additional correlations induced by the measurement process may completely wipe out the genuine cross-correlations caused by the brain dynamics. In the work by Rummel et al. (2007b), the influence of six commonly used EEG references on measures derived from the zero-lag cross-correlation matrix has been studied under different conditions. It was found that the global average reference mainly affects the smallest eigenvalue, such that it is drastically displaced toward smaller values. All other eigenvalues including spacings between them are remarkably insensitive to this particular reference. Therefore, in the present article, we use exclusively the global average reference (for the scalp as well as for the foramen ovale electrodes) and exclude the smallest eigenvalue from the analysis. Hence,

$$
\mathrm{CCS}=\frac{\sum_{i=2}^{M}\left|\widetilde{\lambda}_{i}-\widetilde{\lambda}_{i}^{s}\right| s_{i}}{\sum_{i=2}^{M-1} \widetilde{\lambda}_{i}^{s}+\left(M-\widetilde{\lambda}_{M}^{s}\right)}
$$

However, for exemplary cases, we also tested for a bipolar reference obtaining qualitatively the same results as presented below.

\section{RESULTS}

The presentation of the results is divided into several parts. First, we illustrate the performance of the method with the help of an exemplary case for the whole set of the 12 recordings. We further present the evolution of eigenvalues, the genuine CCS, and the evolution of the power spectra. Finally, we present the outcomes of the whole set of recordings and a statistical validation of the results.

\section{Eigenvalue Spectra for Scalp and Foramen Ovale Electrodes}

In a first step, we calculate the time evolution of the eigenvalues from the recordings with scalp electrodes. Figs. 3A and $3 \mathrm{C}$ display the development of the largest eigenvalue $\widetilde{\lambda}_{19}$ (solid line) and the average of some of the smallest eigenvalues, namely, $\left\langle\lambda^{\text {small }}\right\rangle=\frac{1}{11} \sum_{i=2}^{12} \tilde{\lambda}_{i}$ (dashed line) for seizure 2 of Table 1. Seizure onset and offset are marked by vertical lines.

Before seizure onset, both the largest and the average of the small eigenvalues fluctuate around some mean value. Then, during the seizure, the largest eigenvalue decreases and shows a pronounced minimum while $\lambda^{\text {small }}$ encounters its maximal values. Just before seizure offset, the eigenvalue spectrum spreads out. $\widetilde{\lambda}_{19}$ increases drastically, while $\lambda^{\text {small }}$ decreases and encounters minimal values approximately at second 800 . Approximately 300 seconds after the seizure offset, the eigenvalue spectrum tends to be more narrow again, which can be clearly seen by the increase of $\lambda^{\text {small }}$ at about second 900 to 1,000 . Hence, the width of the eigenvalue spectrum becomes considerably more narrow during the epileptic seizure and its largest extension occurs during the immediate postseizure period.

Because of the argumentation given in The Zero-Lag Correlation Matrix, one can state that the total amount of cross-correlations is small, when $\widetilde{\lambda}_{19}$ takes small and $\lambda^{\text {small }}$ takes large values. In this case, the eigenvalues are narrowly distributed around one. Otherwise, the eigenvalue spectrum is splayed, and the data set contains an elevated amount of cross-correlations. Accordingly, Figs. 3A and $3 \mathrm{C}$ display a pronounced correlation loss during the seizure and a drastic increase of correlations just at the seizure offset. Maximal values of the correlation strength are measured during the immediate postseizure period. As $\widetilde{\lambda}_{19}$ stays on the average at slightly larger values during the whole postseizure period compared with those before seizure onset (and simultaneously $\lambda^{\text {small }}$ stays at somewhat smaller values), one might conclude further that the dynamics stay more correlated even several minutes after seizure offset.

To assess the amount of random correlations, we repeat the analysis with the corresponding data segments of the surrogates. Figs. 3B and 3D display the results. Although, all genuine crosscorrelations are destroyed, the curves show qualitatively similar
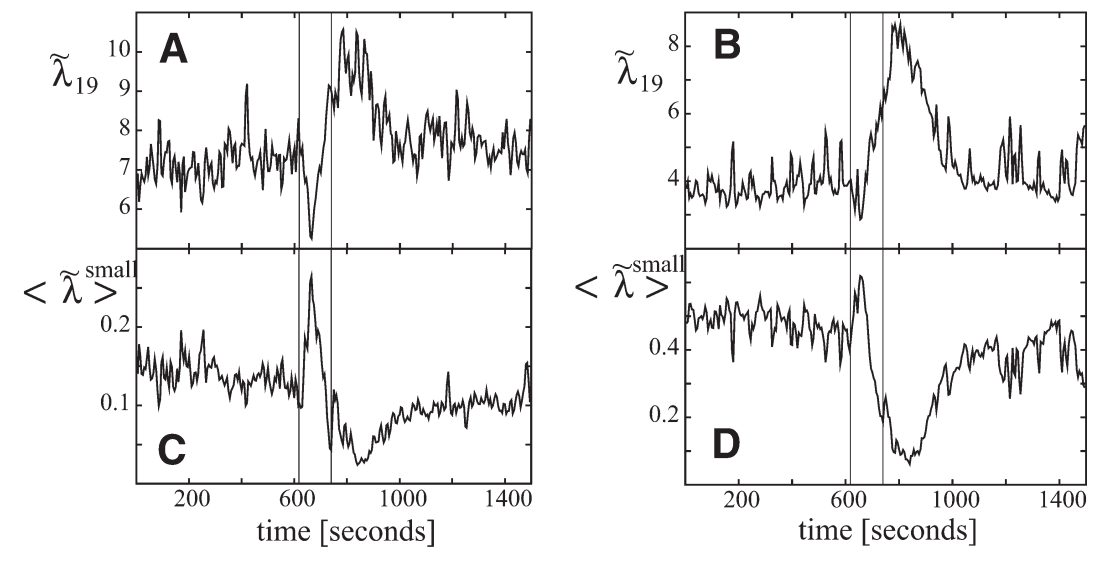

FIG. 3. Evolution of median of eigenvalues of the zero-lag cross-correlation matrix calculated from the 19-channel scalp recording of seizure 2 . The median is taken over the set of eigenvalues obtained for each data segment: A, Median of $\widetilde{\widetilde{\lambda}}_{19}$ obtained from the original data. B, Median of $\widetilde{\lambda}_{19}$ obtained from the surrogates. C, Average of the median of the 11 smallest eigenvalues, determined from the original data. D, Average same as (C) but obtained from surrogates. Seizure onset and offset are indicated by vertical solid lines. 
characteristics. During the seizure, the spectrum gets more narrow, but as in the previous case, a strong repulsion between the largest and the average of the smallest eigenvalues occurs just before seizure offset and even more pronounced during the immediate postseizure period, which lasts approximately 300 seconds.

The present case illustrates that considering the eigenvalues computed from the experimental recording alone may lead to erroneous conclusions. Comparing the results of Figs. 3A and 3B, it remains fully unclear how genuine cross-correlations change during and after seizures.

\section{Estimation of the Genuine \\ Cross-Correlation Strength}

On the grounds of the results presented in Fig. 3, the question arises whether those observations are uniquely caused by incidental contributions or if they are at least partially caused by genuine spatial correlations of neuronal activity. Fig. 4A displays the evolution of the CCS coefficient (Eq. 5) calculated from scalp recordings of seizure 2 discussed so far. Seizure onset and offset are indicated by vertical solid lines.

We note a gradual decrease of the CCS during the seizure, although with some fluctuations. In particular, a pronounced but narrow peak just at seizure offset can be seen. However, such a behavior is not reflected in the overall behavior of all 12 cases (Fig. 5C). The decrease becomes more pronounced after seizure offset, showing a pronounced minimum of approximately 0.02 around second 800 . Thereafter, it increases gradually and recuperates approximately the preseizure value at approximately second 1000 .

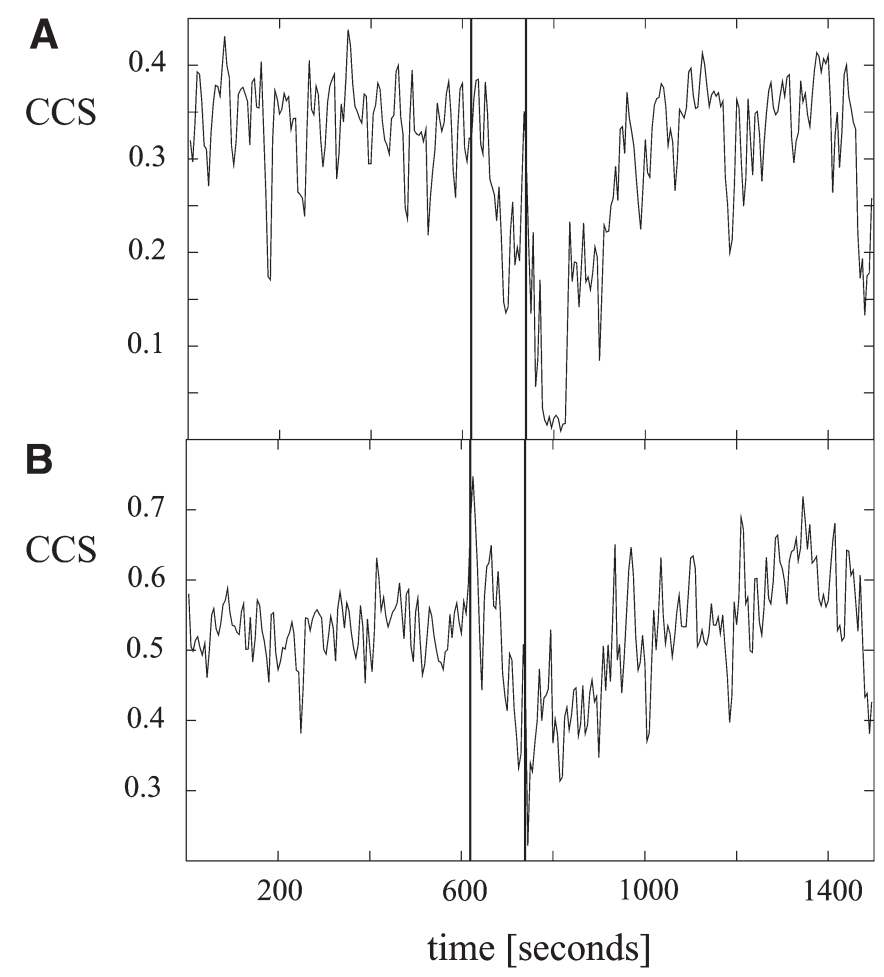

FIG. 4. The CCS value (Eq. 5) obtained from the 19-channel scalp recording of seizure $2(A)$ and from 8 foramen ovale electrodes (B). Seizure onset and offset are indicated by vertical solid lines.
Fig.4B displays the CCS for the case of seizure 2 calculated from the recordings of foramen ovale electrodes. In this case, just at seizure onset, the CCS values encounters an abrupt maximum, but then, as in the case of the extracranial measurement, large fluctuations can be seen during the seizure, again with a decreasing tendency, and it encounters minimal values during the immediate postseizure period. Then, it shows on the average an increasing tendency, and for times larger than second 1,000 fluctuates somehow around the mean preseizure value. Beside of the sudden increase just at seizure onset, the foramen ovale electrodes show qualitatively the same behavior as the scalp electrodes.

\section{Evolution of the Power Spectra}

As we suspect that changes in the frequency content of the signals are the reason for the distribution of eigenvalues of the surrogate data, we calculate the average power within the EEG bands. For visual presentation, the power spectra of each data channel (electrode) were normalized between zero and one. The results for the 19 scalp electrodes of seizure 2 are shown in Fig. 6.

The power in the $\delta$-band decreases slightly during the first half of the seizure but shows a well-pronounced maximum toward seizure offset and during the immediate postseizure period. Then, the power of this band remains on the average at a value slightly below that of the preseizure period.

The power of the $\theta$-band shows a strong increase during the first half of the seizure and a drastic loss of power after seizure offset, which lasts approximately up to second 900 to 950 . Thereafter, the average power in the $\theta$-band recuperates approximately its value of the preseizure period. Higher frequency bands behave in a similar manner. During the seizure, a sharp maximum can be observed, which starts clearly after seizure onset and terminates before seizure offset. However, the region of missing power in the postseizure period is extended for the higher frequency bands. The power in the $\alpha$-band and $\beta$-band remains low over the whole time course after the seizure. The time evolution of the power of the different frequency bands of the foramen ovale recordings is qualitatively similar to that of the scalp recordings of seizure 2 .

\section{Relative Changes of the Genuine Cross-Correlation Strength}

We turn now to the statistical evaluation of relative changes of the CCS for all 12 seizures, as described above. The results for the scalp and for the foramen ovale electrodes are summarized in Figs. $5 \mathrm{~A}$ and $5 \mathrm{~B}$, which shows median CCS values for the 4 segments of the scalp and the foramen ovale recordings, respectively, while Figs. 5C and 5D shows the evolution of the median value of all 12 seizures for the seizure period, respectively, for scalp and foramen ovale recordings. To obtain the results of Figs. $5 \mathrm{C}$ and $5 \mathrm{D}$, we normalized the duration of all 12 seizures to the same unit length.

The results obtained for EEGs recorded with scalp electrodes (Figs. 5A and 5C) reflect qualitatively the behavior illustrated by the example of seizure 2. The CCS is slightly decreased during the seizure period and reaches a pronounced minimum in the immediate postseizure period P1 (Fig. 5A). Only a few minutes after seizure offset (segment P2), the amount of cross-correlations attains an average value marginally below that of the preseizure period. The inspection of the seizure period reveals that the median CCS values of the first half of the seizure are on the average larger than those of the second half (Fig. 5C). This confirms the observations of seizure 2 (Fig. 4A), where on the average, a continuous decrease of the 

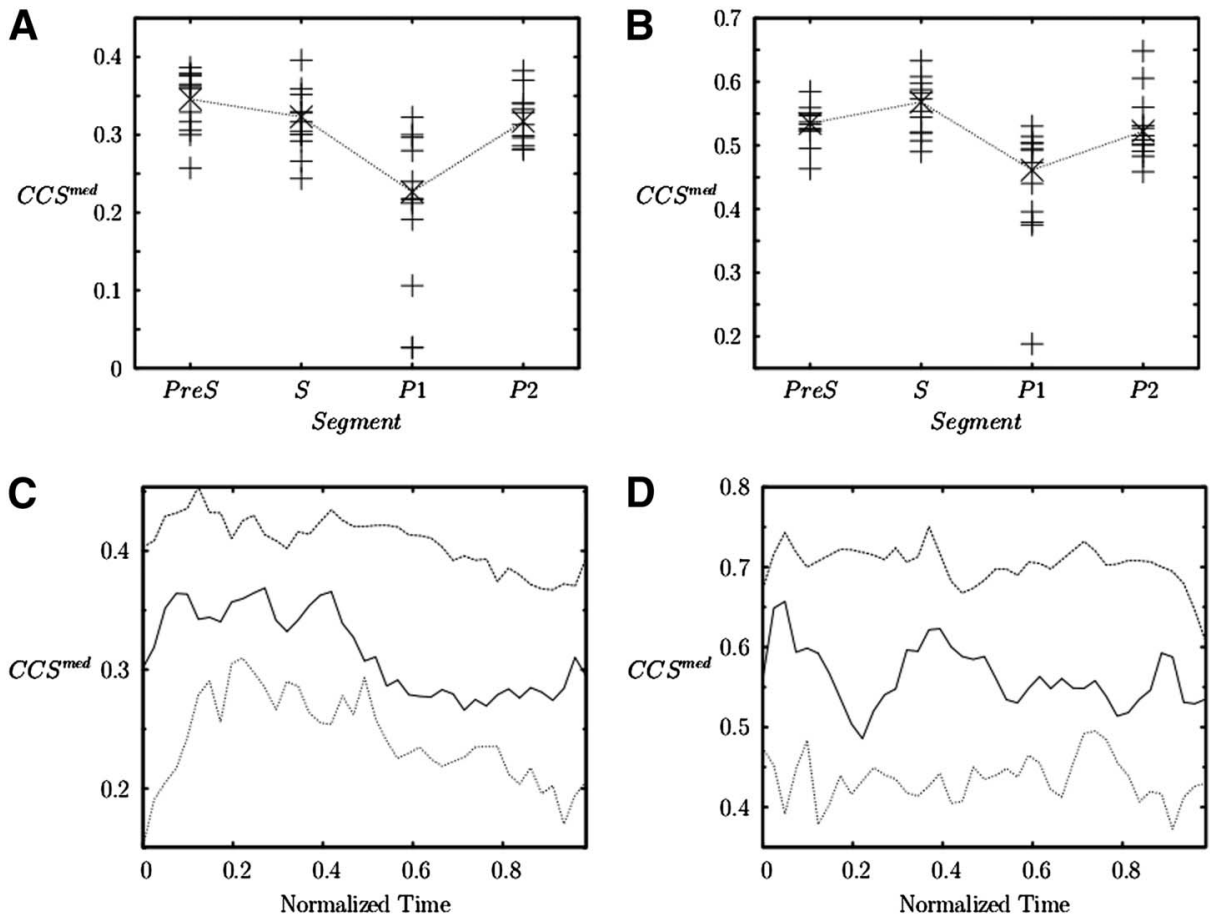

FIG. 5. Median CCS values of each segment for the scalp recording (A) and the foramen ovale electrodes (B). The + sign indicates the median values of each seizure and the $x$ symbol marks the median of all recordings. The dashed line connects the median values of all recordings for a better visibility. To improve the visibility, we connected the $\times$ symbols with a dashed line. C, Evolution of the median CCS value during the (normalized) seizure period (solid line). The median is taken from the CCS values of the 12 seizures. The dashed line markers the $75 \%$ confidence interval. D, Same as (C) for the foramen ovale electrodes . average CCS value has been observed during seizure and immediate postseizure period.

For the foramen ovale electrodes (Fig. 5B), the CCS value of the seizure epoch is slightly larger than in segment PreS. For later periods, the behavior is qualitatively similar to that of the scalp recordings. The median value for $\mathrm{P} 2$ is slightly lower than that of segment PreS, and minimal values are measured for segment P1.

However, Fig. 5D does not show the slight decrease of genuine correlations during the seizure period of the scalp electrodes (it neither shows any kind of increasing tendency). Now the median values fluctuate somehow around some constant value.

In view of the exemplary results for the power spectra, the question arises whether the evolution of the CCS alters for different frequency bands. Therefore, we repeated the same analysis for band pass-filtered signals. The results for the scalp electrodes are drawn in the left and those for the foramen ovale in the right column of Fig. 7. The $P$ values of a two-sided median test for the transitions from on segment to another are given in Table 2.

\section{Scalp Electrodes}

The results obtained for the $\delta$-band, $\theta$-band, and $\alpha$-band are qualitatively similar for the four previously defined segments. In all cases, a gradual decrease of the CCS can be seen for segment S and $\mathrm{P} 1$, that is, for the whole seizure and the immediate postseizure period. Minimal values are always detected for segment P1. This behavior is most pronounced for frequencies below $12.5 \mathrm{~Hz}$. For the $\beta$-band, the situation is different. Here, the median value of CCS is maximal for segment $\mathrm{S}$ while, like in the case of lower frequencies, minimal values are detected for segment P1.

\section{Foramen Ovale Electrodes}

The evolution of the CCS from the preseizure to the postseizure period is qualitatively different to that obtained by the extracranial measurement. Now the maximum median value of the $\mathrm{CCS}$ is attained for the seizure segment $\mathrm{S}$ for all frequency bands.
The behavior of the minimal value is not such uniform. For the $\delta$-band, it is given for the immediate postseizure period P1, while for all other cases, the preseizure period is characterized by minimal CCS values. However, for all cases, the median values for segment $\mathrm{P} 1$ are significantly lower than those of the period S.

To quantify the significance of the changes of the 12 seizures between the first three and all four segments, we apply a Friedman test.

According to the Friedman test, the null hypothesis that the CCS values of the three segments of the scalp recordings are statistically equivalent can be rejected, with the exception of the $\delta$-band, that is, beside of the slowest frequency, the changes from preseizure to immediate postseizure activity are significant.

For foramen ovale electrodes, in the $\alpha$-band, the null hypothesis cannot be rejected. Considering all four segments, the Friedman test cannot reject the null hypothesis in all cases for the foramen ovale electrodes and for the $\delta$-band and $\alpha$-band in case of the scalp recordings. These results are caused by transitions to the postseizure period P2. However, in most cases, the CCS values of the first three segments show significant differences. By looking at each pair of segments separately via a two-sided median test, one gains a more detailed picture (Table 3). According to the results presented in Table 3, the null hypothesis can be rejected for all cases of the broad band data, on a $1 \%$ significance level with one exception. The CCS values of postseizure segment P2 seem to be statistically equivalent to those of the seizure period.

Furthermore, for extracranial recordings, the segments PreS and $\mathrm{P} 2$ are considered equivalent in the $\delta$-band, $\alpha$-band, and $\beta$-band, while the segments S and P2 are not significantly different for the $\delta$-band and $\theta$-band. For the semi-invasive foramen ovale electrodes for all pairs of segments of the broad band case as well as the $\delta$-band, the null hypothesis of equivalence can be rejected, while segments PreS and P1 show statistically similar median values for the $\alpha$-band and $\beta$-band and equivalently the segments PreS and $\mathrm{S}$ for the $\theta$-band. Furthermore, there are no significant changes between the two postseizure periods when considering the $\beta$-band, and the seizure 


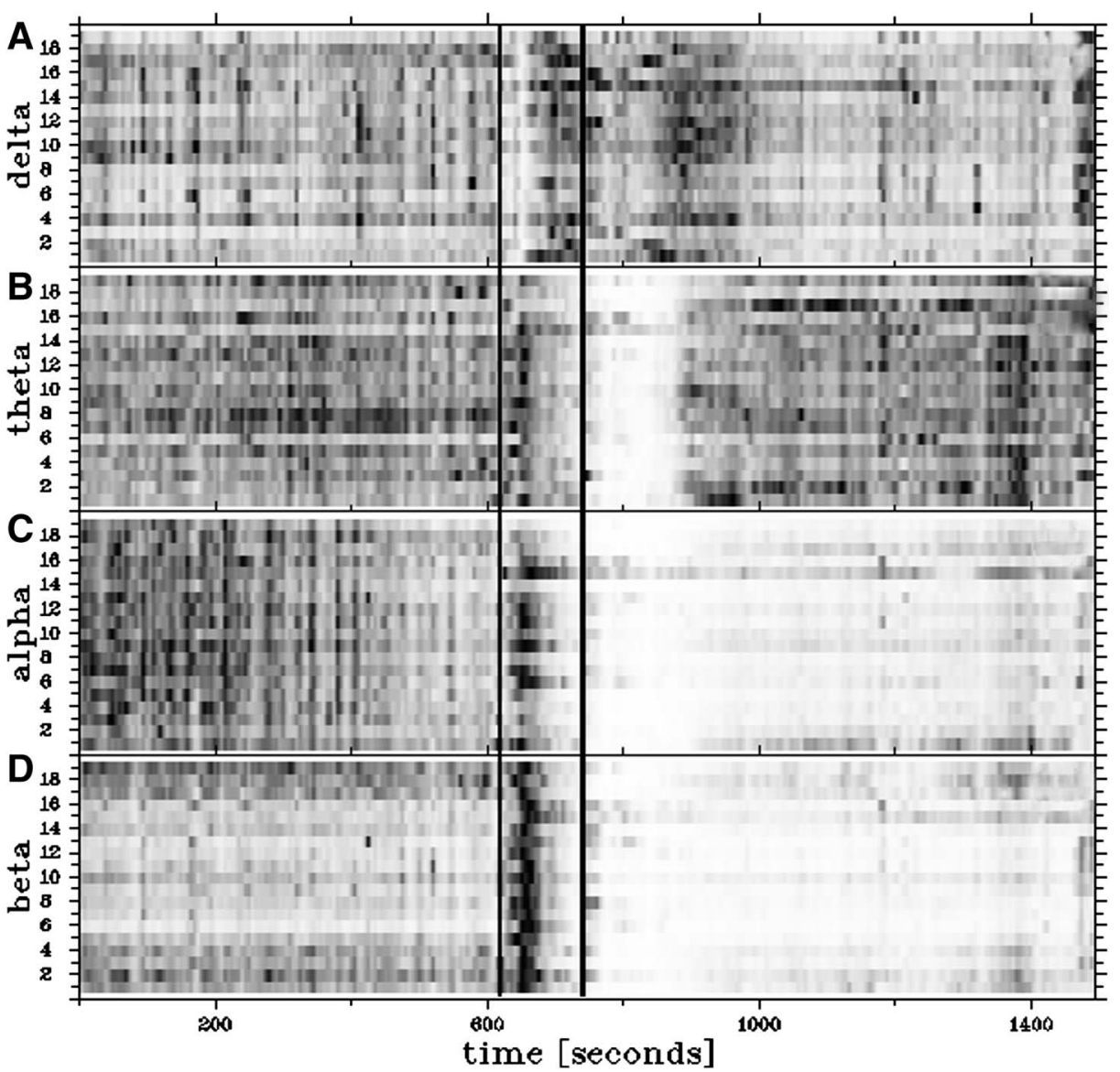

FIG. 6. Normalized power of the different frequency band of the scalp recording of seizure 2 . The evolution of the power averaged over the $\delta$-band (A), $\theta$-band (B), $\alpha$-band (C), and $\beta$-band (D) are shown. The numbering of the electrodes is as follows: 1-F3, 2-F4, 3-C3, 4-C4, 5-P3, 6-P4, 7-O1, 8-O2, 9-F7, 10-F8, 11-T3, 12-T4, 13-T5, 14-T6, 15-F9, 16-F10, $17-F z, 18-C z$, and 19-Pz. Seizure onset and offset are indicated by vertical solid lines. Gray scale coding: black color corresponds to a relative power equal to one, and white corresponds to zero. segment $\mathrm{S}$ is not significantly different to the postseizure period P2 for the $\alpha$-band.

In summary, the changes of CCS values between the segments preictal, ictal, and immediate postictal are significant for all cases when scalp recordings are considered. Only some of the transitions to the postictal segment do not cause significant changes of the CCS values. For the broad band case and for frequencies below $12.5 \mathrm{~Hz}$, this means a continuous significant decrease of genuine cross-correlations. For foramen ovale recordings, only for broad band data and the $\delta$-band, CCS values of the immediate postseizure period encounter significantly smaller values as those of the preseizure period. For higher frequency bands, there is no significant difference between the CCS values considering a $1 \%$ significance level. However, the observed increase of genuine cross-correlation during the seizure period is significant for broad band data and for all frequency bands (transition PreS-S and S-P1).

\section{DISCUSSION}

Central objects of the present study are the eigenvalues of the zero-lag cross-correlation matrix. By just considering the width of the eigenvalue distribution, we observe a loss of total crosscorrelations during the seizure followed by a drastic increase in the total strength of correlations at seizure offset (Figs. 3A and 3C). These findings are in accordance with the results obtained by Bartolomei et al. (2004), Guye et al. (2006), Schindler et al. (2007b), and Wendling et al. (2003), where occasionally a slight correlation loss at seizure onset has been observed but in any case enhanced correlation of the brain dynamics measured by intracranial recordings toward seizure offset. However, in this case, no distinction between random and genuine cross-correlation is made.

The observed variation of the widths of the eigenvalue spectra (Figs. 3A and 3C) coincides with characteristic changes of the power spectra as demonstrated by the example of seizure 2 (Fig. 6). During the seizure, the $\delta$-power is slightly suppressed and increases considerably in the immediate postseizure epoch. The $\theta$-power behaves exactly opposite to that: it increases during seizure and decreases at seizure offset, a behavior even more pronounced in the $\alpha$-band and $\beta$-band. This alternation of the power spectra has a direct influence on the amount of random correlations, which enter to matrix (Eq. 2) and provoke characteristic changes of the eigenvalue distribution (Figs. 3B and 3D). A relative increase (decrease) of the $\delta$-power promotes a broadening (narrowing) of the eigenvalue distribution while a relative enhancement (suppression) of higher frequency bands cause a narrowing (broadening) of the spectrum.

However, conclusions drawn uniquely from the eigenvalues may change drastically, when the influence of the frequency content of the signals is accounted for. The measure (Eq. 5) captures exclusively the strength of genuine zero-lag cross-correlations and is designed such that also subtle correlations between a few data channels are detected (Müller et al., 2008a). According to this measure, we gave clear evidence that seizures are characterized by a reduced amount of genuine zero-lag cross-correlations with a further decrease during the immediate postseizure period. This is at least true for frequencies below $12.5 \mathrm{~Hz}$ when scalp recordings are considered (see Figs. 5A and 7; Tables 2 and 3). 

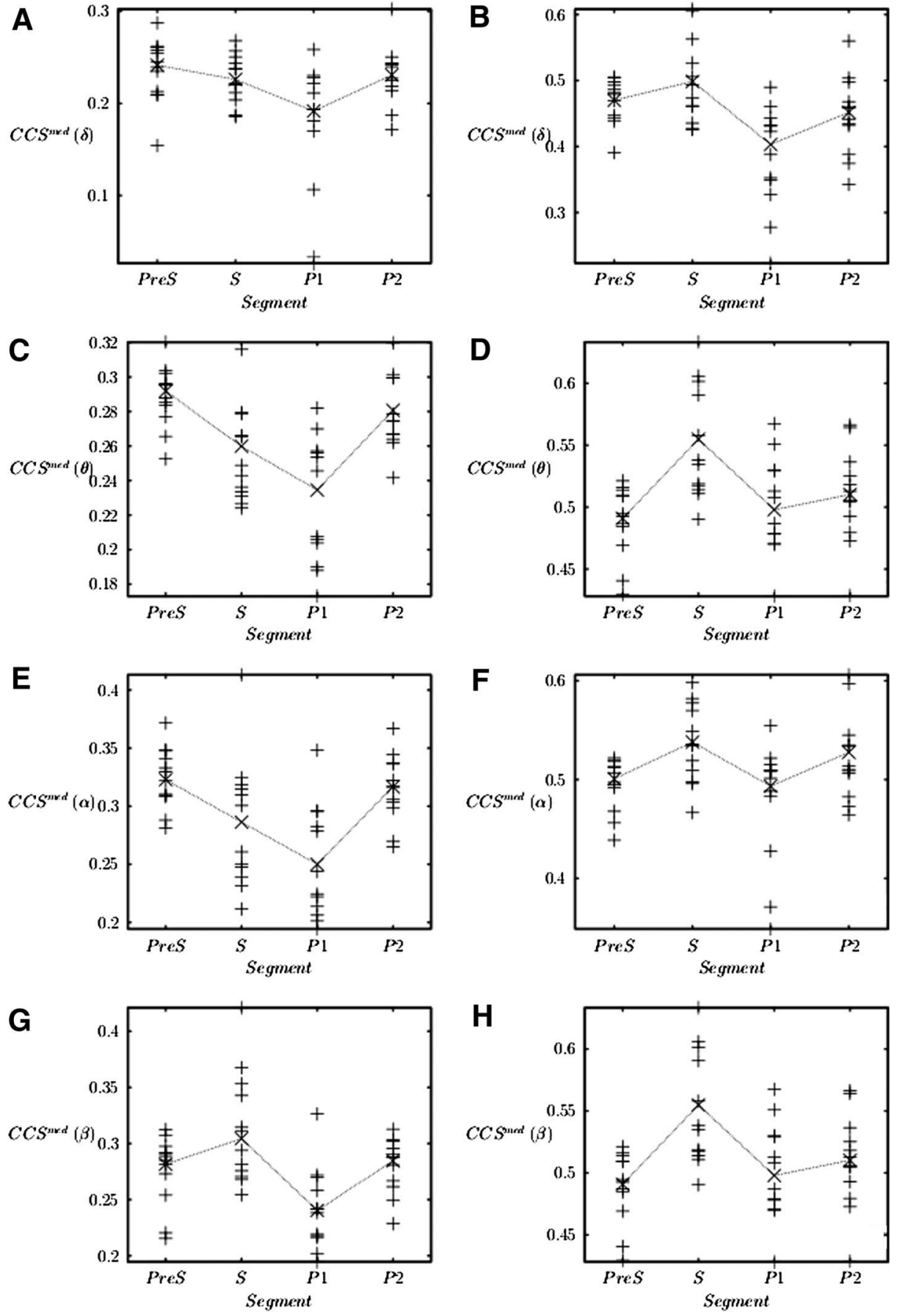

FIG. 7. Median CCS for the scalp recording ( $A, C, E$, and $\mathbf{G}$ ) and the foramen ovale electrodes (B, D, F, and $\mathrm{H})$. The $<\mathrm{CCS}>$ for band passfiltered signals according to the EEG bands. $\delta$-band ( $A$ and $B$ ), $\theta$-band ( $C$ and $\mathbf{D}), \alpha$-band (E and $F)$, and $\beta$-band $(\mathbf{G}$ and $\mathbf{H})$. Symbols are chosen as in Figs. 5A and 5B. The dashed line connects the median values of all recordings for a better visibility.
In view of the results for the CCS, this implies that the increased power of the $\theta$-band and $\alpha$-band during the seizure and the increased power of the $\delta$-band in the immediate postseizure epoch is essentially uncorrelated in terms of zero-lag correlations. Most prominent in this context is the increase of $\delta$ activity and the simultaneous decrease of power in the higher frequency bands starting before seizure offset. During the same period, the largest eigenvalues (calculated from the surrogates and the original data) repel strongly with most of the smallest eigenvalues, that is, the eigenvalue distribution becomes broader, clearly indicating an increase of the total correlation strength. As the CCS value shows a well-pronounced minimum within the same epoch (Figs. 4A, 5A and 7), the increase in total correlation is uniquely caused by random contributions. Furthermore, the sharp maximum of higher frequency power during seizure (Fig. 6) neither increases the amount of genuine nor that of random correlations. Hence, decorrelation is observed in terms of CCS for a relatively broad frequency band of approximately 0.5 to $12.5 \mathrm{~Hz}$. This result might be interpreted as a functional segregation of brain dynamics. In contrast, higher frequency components ( $\beta$-band) become more correlated during seizure (Fig. 7G). However, despite low-pass filtering at $20 \mathrm{~Hz}$, we cannot exclude with certainty that at least part of this effect might be caused by muscle artifacts. 
TABLE 2. $\quad P$ Values of the Two-Sided Friedman Test for the First Three Segments Pre, S, and P1 (Denoted by F3) and All Four Segments Pre, S, P1, and P2 (Denoted by F4)

\begin{tabular}{lll}
\hline Case $\backslash$ Number of Segments & F3 & F4 \\
\hline Scalp electrodes & & \\
Broad band & 0.0023 & 0.0031 \\
$\delta$ & $\mathbf{0 . 0 9}$ & $\mathbf{0 . 1 3 5}$ \\
$\theta$ & 0.001 & 0.0003 \\
$\alpha$ & 0.01 & $\mathbf{0 . 0 1 3}$ \\
$\beta$ & 0.004 & 0.002 \\
Foramen ovale & & \\
Broad band & 0.0003 & $\mathbf{0 . 0 2}$ \\
$\delta$ & 0.004 & $\mathbf{0 . 0 3}$ \\
$\theta$ & 0.002 & $\mathbf{0 . 6 1}$ \\
$\alpha$ & $\mathbf{0 . 0 9}$ & $\mathbf{0 . 1 1}$ \\
$\beta$ & 0.002 & $\mathbf{0 . 0 2}$ \\
\hline
\end{tabular}

Transitions that are not significant on a $1 \%$ significance level are marked with bold face numbers.

Our observation is consistent with a study of intrahemispheric and interhemispheric propagation of ictal discharges of complex partial seizures (Lieb et al., 1987). There, coherence and phase analysis have been applied to 28 recordings of 10 patients to reveal the emergence of linear relationships between different brain regions. The authors report that although strong intrahemispheric coherence reliably emerge in both the epileptogenic and nonepileptogenic hemisphere during seizure onset and collateral spread, these relationships were usually not observed for interhemispheric comparison. Also, the application of nonlinear techniques failed to prove the emergence of any relationships between left and right hemispheres (Mars et al., 1985). A missing functional coupling between the hemispheres and therefore a reduced long-range functional coupling surely promote the loss of genuine correlation strength, as observed in the present study.

A further support for the observation of a correlation loss at seizure onset even for higher frequencies has been reported by Wendling et al. (2003), where recordings from intracranial electrodes implanted in the prefrontal or frontal region have been analyzed. In this analysis, only frequencies between 24 and $128 \mathrm{~Hz}$ have been studied by computing the Pearson coefficient. The authors reported a significant decorrelation (mainly between 60 and $90 \mathrm{~Hz}$, i.e., a frequency band not considered in the present work) during the phase of rapid discharges, while the power in this frequency band simultaneously increases. Then, an abnormally high recoupling, when seizure develops, was observed with highest values toward seizure offset. However, for higher frequency bands, the role of random correlations diminishes because here the time window used to calculate the Pearson coefficient gets large in comparison with the period of the oscillations (Rummel et al., 2010). Also, in the present study, we observed elevated values for the genuine cross-correlations for the $\beta$-band for the seizure and postseizure period measured by extracranial and intracranial recordings (Figs. $7 \mathrm{G}$ and $7 \mathrm{H}$ ), although, as already mentioned, we cannot exclude the influence of muscle artifacts for the $\beta$-band, especially during seizure.

Foramen ovale measurements cover a much smaller area than several grid or strip electrodes placed directly onto (selected areas of) the cortex or extracranial measurements that cover the whole scalp surface. Furthermore, shorter time scales as defined by higher frequency components correspond to smaller spatial scales, namely, wavelengths. This may explain why we found a significant increase in genuine cross-correlations during the seizure for broad band data (Fig. 5B), which is mostly generated by activity in the $\alpha$-band and $\beta$-band (Figs. 7F and 7H) but not by lower frequencies of the $\delta$-band. Also, the minimal correlation values during the immediate postseizure period are caused mainly by frequencies of the $\delta$-band and $\theta$-band.

When considering zero-lag cross-correlations, one might expect volume conduction to affect the results, in particular during high-amplitude seizure activity. However, it is plausible to assume that volume conduction would increase the amount of zero-lag crosscorrelations, particularly during the seizures, when high-amplitude signals are generated. Instead, we observed a clear tendency for a decorrelation toward seizure offset (Fig. 5C) for the scalp recordings. Hence, if volume conduction has an effect in the cases considered in this study, we expect that it diminished the significance of our main findings (decrease of CCS during seizure and immediate postseizure period).

Finally, we come back to the hypothesis that a more correlated neuronal activity might be an emergent self-regulatory mechanism for seizure offset. If, as argued in Schindler et al. (2007a, 2007b), the brain generates a more correlated dynamics, which then leads to seizure offset, our results suggest that this goal is reached on macroscopic

TABLE 3. $\quad P$ Values of the Two-Sided Median Test of the CCS Values for the Transition Between Different Segments for the Scalp (Above) and Foramen Ovale Electrodes (Below)

\begin{tabular}{|c|c|c|c|c|c|c|}
\hline Case/Transition & Pre-S & Pre-P1 & Pre-P2 & S-P1 & S-P2 & P1-P2 \\
\hline Broad band & $0.1 \mathrm{E}-4$ & $0.1 \mathrm{E}-10$ & $0.1 \mathrm{E}-10$ & $0.1 \mathrm{E}-10$ & 0.15 & $0.1 \mathrm{E}-10$ \\
\hline$\delta$ & $0.4 \mathrm{E}-3$ & $0.1 \mathrm{E}-10$ & 0.02 & $0.2 \mathrm{E}-4$ & 0.32 & $0.6 \mathrm{E}-7$ \\
\hline$\alpha$ & $0.1 \mathrm{E}-10$ & $0.1 \mathrm{E}-10$ & 0.06 & $0.4 \mathrm{E}-3$ & $0.5 \mathrm{E}-5$ & $0.1 \mathrm{E}-10$ \\
\hline$\beta$ & $0.9 \mathrm{E}-3$ & $0.1 \mathrm{E}-10$ & 0.15 & $0.1 \mathrm{E}-10$ & $0.1 \mathrm{E}-3$ & $0.1 \mathrm{E}-10$ \\
\hline \multicolumn{7}{|l|}{ Foramen ovale } \\
\hline$\theta$ & 0.43 & $0.1 \mathrm{E}-10$ & $0.9 \mathrm{E}-6$ & $0.1 \mathrm{E}-3$ & 0.0025 & $0.1 \mathrm{E}-10$ \\
\hline$\alpha$ & $0.2 \mathrm{E}-8$ & 0.73 & $0.3 \mathrm{E}-7$ & $0.1 \mathrm{E}-10$ & 0.019 & $0.5 \mathrm{E}-5$ \\
\hline$\beta$ & $0.1 \mathrm{E}-10$ & 0.17 & $0.1 \mathrm{E}-4$ & $0.1 \mathrm{E}-10$ & $0.1 \mathrm{E}-10$ & 0.03 \\
\hline
\end{tabular}

\footnotetext{
Transitions that are not significant on a $1 \%$ significance level are marked with bold face numbers.
} 
scale (whole scalp) only in a statistical sense, at least when frequency components below $12.5 \mathrm{~Hz}$ are considered. For those slow components, we find that only the random but not the genuine cross-correlations increase around seizure offset. However, the amount of the random contributions depends not only on the relative power of the frequency bands but also on the choice of T (i.e., the length of the data segment used for the construction of the Pearson coefficients $C_{i j}$ ), as argued in the discussion of Fig. 1 (Müller et al., 2008a; Rummel et al., 2010). Therefore, we do not consider the evolution of random correlations in this article. Alternatively, one might hypothesize that the successive decrease of the amount of genuine zero-lag cross-correlations and therefore the functional disintegration of different brain areas on large spatial scales for low frequency activity is the responsible mechanism for seizure offset. At least, seizure offset is accompanied by a suppression of genuine zero-lag cross-correlations.

However, such questions cannot be conclusively answered in the present study but require further investigations. Furthermore, we stress that the fact that zero-lag cross-correlations decrease does not necessarily implies that the brain dynamics diminishes its collectivity if non-zero lags or nonlinear interactions are considered. It is also conceivable that some oscillatory dynamical state, as, for example, detected during sleep (Massimini et al., 2004; Olbrich and Ackermann, 2005; Steriade, 2006), produce almost constant phase differences of the signals measured by different scalp electrodes, such that zero-lag cross-correlations decrease on the average, which might be another interesting aspect for a subsequent study. Finally, we propose a similar study with intracranial data similar to those used in Schindler et al. (2007b) to investigate whether a similar characteristic decrease of genuine cross-correlations occur also within a spatial scale defined by the dimensions of a grid of electrodes or if our results for the foramen ovale electrodes can be reproduced.

\section{ACKNOWLEDGMENTS}

The authors appreciate the constructive criticism and suggestions of the anonymous referees.

\section{REFERENCES}

American EEG Society. Guideline seven: a proposal for standard montages to be used in clinical EEG. J Clin Neurophysiol 1986;3:158-165.

Anderson TW. An introduction to multivariate statistical analysis. New York, NY: Wiley, 2003.

Ansari-Asl K, Senhadji L, Bellanger JJ, Wendling F. Quantitative evaluation of linear and nonlinear methods characterizing interdependencies between brain signals. Phys Rev E Stat Nonlin Soft Matter Phys 2006;74:031916.

Arnhold J, Grassberger P, Lehnertz K, Elger CE. A robust method for detecting interdependencies: application to intracranially recorded EEG. Phys Nonlinear Phenom 1999;134:419-430.

Babloyantz A, Destexhe A. Low-dimensional chaos in an instance of epilepsy. Proc Natl Acad Sci U S A 1986;83:3513-3517.

Babloyantz A, Salazar JM, Nicolis C. Evidence for chaotic dynamics of brain activity during the sleep cycle. Phys Lett A 1985;111:152-156.

Baier G, Müller M, Stephani U, Muhle H. Characterizing correlation changes of complex pattern transitions: the case of epileptic activity. Phys Lett A 2007;363:290-296.

Bartolomei F, Wendling F, Bellanger JJ, et al. Neural networks involving the medial temporal structures in temporal lobe epilepsy. Clin Neurophysiol 2001;112:1746-1760.

Bartolomei F, Wendling F, Régis J, et al. Pre-ictal synchronicity in limbic networks of mesial temporal lobe epilepsy. Epilepsy Res 2004;61:89-104.

Bhattacharya J. Reduced degree of long-range phase synchrony in pathological human brain. Acta Neurobiol Exp (Wars) 2001;61:309-318.

Casdagli M. Chaos and deterministic versus stochastic non-linear modelling. $J R$ Statist Soc B 1992;54:303-328.

Chavez M, Besserve M, Adam C, Martinerie J. Towards a proper estimation of phase synchronization from time series. $J$ Neurosci Methods 2006;154:149-160.

Conlon T, Ruskin HJ, Crane M. Cross-correlation dynamics in financial time series. Phys Stat Mech Appl 2009a;388:705-714.
Conlon T, Ruskin HJ, Crane M. Seizure characterization using frequency-dependent multivariate dynamics. Comput Biol Med 2009b;39:760-767.

Guye M, Régis J, Tamura M, et al. The role of corticothalamic coupling in human temporal lobe epilepsy. Brain 2006;129:1917-1928.

Hegger R, Kantz H, Schreiber T. Practical implementation of nonlinear time series methods: the TISEAN package. Chaos 1999;9:413-435

Iasemidis LD, Shiau DS, Chaovalitwongse W, et al. Adaptive epileptic seizure prediction system. IEEE Trans Biomed Eng 2003;50:616-627.

Jerger KK, Netoff TI, Francis JT, et al. Comparison of methods for seizure detection. In: Milton J, Jung P, eds. Epilepsy as a dynamic disease. Berlin: Springer-Verlag, 2002:237-246.

Kantz H, Schreiber T. Nonlinear time series analysis. Cambridge: Cambridge University Press, 1997. Cambridge nonlinear science series. Vol 7.

Keener J, Sneyd J. Mathematical physiology. New York, NY: Springer, 1998

Kreuz T, Mormann F, Andrzejak RG, et al. Measuring synchronization in coupled model systems: a comparison of different approaches. Phys Nonlinear Phenom 2007;225:29-42.

Lehnertz K, Elger CE. Can epileptic seizures be predicted? Evidence from nonlinear time series analysis of brain electrical activity. Phys Rev Lett 1998;80:5019-5022.

Lehnertz K, Arnhold L, Grassberger P, Elger CE. Chaos in the brain? Singapore; World Scientific, 2000.

Lieb JP, Hoque K, Skomer CE, Song XW. Inter-hemispheric propagation of human mesial temporal lobe seizures: a coherence/phase analysis. Electroencephalogr Clin Neurophysiol 1987;67:101-119.

Mann HB, Whitney DR. On a test of whether one or two random variables is stochastically larger than the other. Ann Math Stat 1947;18:50-60.

Mars NJ, Lopes da Silva FH. Propagation of seizure activity in kindled dogs. Electroencephalogr Clin Neurophysiol 1983;56:194-209.

Mars NJ, Thompson PM, Wilkus RJ. Spread of epileptic seizure activity in humans. Epilepsia 1985;26:85-94.

Massimini M, Huber R, Ferrarelli F, et al. The sleep slow oscillation as a traveling wave. J Neurosci 2004;24:6862-6870.

Mormann F, Lehnertz K, David P, Elger CE. Mean phase coherence as a measure for phase synchronization and its application to the EEG of epilepsy patients. Phys Nonlinear Phenom 2000;144:358-369.

Mormann F, Kreuz T, Rieke C, et al. On the predictability of epileptic seizures. Clin Neurophysiol 2005;116:569-587.

Morrison DF. Multivariate statistical methods. 4th ed. Belmont: Thomson, 2005.

Müller M, Baier G, Galka A, et al. Detection and characterization of changes of the correlation structure in multivariate time series. Phys Rev E Stat Nonlin Soft Matter Phys 2005;71:046116.

Müller M, López Jiménez Y, Rummel C, et al. Localized short-range correlations in the spectrum of the equal-time correlation matrix. Phys Rev E Stat Nonlin Soft Matter Phys 2006;74:041119.

Müller M, Baier G, Rummel C, Schindler K. Estimating the strength of genuine and random correlations in non-stationary multivariate time series. Eur Phys Lett 2008a;84:10009.

Müller M, Baier G, Rummel C, et al. A multivariate approach to correlation analysis based on random matrix theory. In: Schelter B, Timmer J, Schulze-onhage A, eds. Seizure prediction in epilepsy: from basic mechanisms to clinical applications. 1st ed. Darmstadt, Germany: Wiley-CH 2008b:209-226.

Nan X, Jinghua X. The fractal dimension of EEG as a physical measure of conscious human brain activities. Bull Math Biol 1988;50:559-565.

Netoff TI, Schiff SJ. Decreased neural synchronization during experimental seizures. J Neurosci 2002;22:7297-7307.

Netoff TI, Clewley R, Arno S, et al. Epilepsy in small-world networks. J Neurosci 2004;24:8075-8083.

Olbrich E, Ackermann P. Analysis of oscillatory pattern in the human sleep EEG using a novel detection algorithm. J Sleep Res 2005;14:337-346.

Palus M. Nonlinearity in normal human EEG: cycles, temporal asymmetry, nonstationarity and randomness, not chaos. Biol Cybern 1996;75:389-396.

Pijn JP, Lopes da Silva FH. Propagation of electrical activity: nonlinear associations and time delays between EEG signals. In: Zschocke S, Speckmann E,-J, eds. Basic mechanisms of the EEG brain dynamics. Boston, MA: Birkhäuser, 1993:41-61

Pijn JP, van Neerven J, Noest A, Lopes da Silva FH. Chaos or noise in EEG signals; dependence on state and brain site. Electroencephalogr Clin Neurophysiol 1991;79:371-381.

Pijn JP, Velis DN, van der Heyde MJ, et al. Nonlinear dynamics of epileptic seizures on basis of intracranial EEG recordings. Brain Topogr 1997;9:249-270.

Pikovsky A, Rosenblum M, Kurths J. Synchronization: a universal concept in nonlinear science. Cambridge, UK: Cambridge University Press, 2001.

Plerou V, Gopikrishnan P, Rosenow B, et al. Random matrix approach to cross correlations in financial data. Phys Rev E Stat Nonlin Soft Matter Phys 2002;65:066126.

Ponten SC, Bartolomei F, Stam CJ. Small-world networks and epilepsy: graph theoretical analysis of intracerebrally recorded mesial temporal lobe seizures. Clin Neurophysiol 2007;118:918-927. 
Press WH, Teukolsky SA, Vetterling WT. Numerical recipes: the art of scientific computing. Cambridge, MA: Cambridge University Press, 2007.

Rapp PE, Zimmerman ID, Albano AM, et al. Dynamics of spontaneous neural activity in the simian motor cortex: the dimension of chaotic neurons. Phys Lett A 1985;110:335-338.

Rosenblum M, Pikovsky AJ, Kurths J. Synchronization approach to analysis of biological systems. Fluct Noise Lett 2004;4:L53-L62.

Rummel C. Quantification of intra- and inter-cluster relations in nonstationary and noisy data. Phys Rev E Stat Nonlin Soft Matter Phys 2008;77:016708.

Rummel C, Baier G, Müller M. Automated detection of time-dependent crosscorrelation clusters in nonstationary time series. Eur Phys Lett 2007a;80:68004.

Rummel C, Baier G, Müller M. The influence of static correlations on multivariate correlation analysis of the EEG. J Neurosci Methods 2007b;166:138-157.

Rummel C, Müller M, Baier G, et al. Analyzing spatio-temporal patterns of genuine cross-correlations. J Neurosci Methods 2010;191:94-100.

Schindler K, Elger CE, Lehnertz K. Increasing synchronization may promote seizure termination: evidence from status epilepticus. Clin Neurophysiol 2007a;118:1955-1968.

Schindler K, Leung H, Elger CE, Lehnertz K. Assessing seizure dynamics by analysing the correlation structure of multichannel intracranial EEG. Brain 2007b;130:65-77.

Schreiber T, Schmitz A. Surrogate time series. Phys Nonlinear Phenom 2000; $142: 346-382$.

Siegel S. Non-parametric statistics for the behavioral sciences. 2nd ed. New York, NY: Mc Graw Hill, 1988.

Siegfried J, Wieser HG, Stodieck SRG. Foramen ovale electrodes: a new technique enabling presurgical evaluation of patients with mesiobasal temporal lobe seizures. Appl Neurophysiol 1985;48:408-417.

Skarda CA, Freeman WJ. How brains make chaos in order to make sense of the world. Behav Brain Sci 1987;10:161-195.
Sporns O, Zwi JD. The small world of the cerebral cortex. Neuroinformatics 2004;2:145-162.

Stam CJ. Nonlinear dynamical analysis of EEG and MEG: review of an emerging field. Clin Neurophysiol 2005;116:2266-2301.

Stam CJ, van Dijk BW. Synchronization likelihood: an unbiased measure of generalized synchronization in multivariate data sets. Phys Nonlinear Phenom 2002;163:236-251.

Stepien RA. Testing for non-linearity in EEG signals of healthy subjects. Acta Neurobiol 2002;62:277-281.

Steriade M. Grouping of brain rhythms in corticothalamic systems. Neuroscience 2006;137:1087-1106

Theiler J. On the evidence for low-dimensional chaos in an epileptic electroencephalogram. Phys Lett A 1995;196:335-341.

Theiler J, Rapp PE. Re-examination of the evidence for low-dimensional, nonlinear structure in the human electroencephalogram. Electroencephalogr Clin Neurophysiol 1996;98:213-222.

Topolnik L, Steriade M, Timofeev I. Partial cortical deafferentation promotes development of paroxysmal activity. Cereb Cortex 2003;13:883-893.

Wendling F, Bartolomei F, Bellanger JJ, Chauvel P. Interpretation of interdependencies in epileptic signals using a macroscopic physiological model of the EEG. Clin Neurophysiol 2001;112:1201-1218.

Wendling F, Bartolomei F, Bellanger JJ, et al. Epileptic fast intracerebral EEG activity: evidence for spatial decorrelation at seizure onset. Brain 2003;126:1449-1459.

Wilcoxon F. Individual comparison by ranking methods. Biometrics Bull 1945;1:80-83.

$\mathrm{Xu} \mathrm{L}$, Chen Z, Hu K, et al. Spurious detection of phase synchronization in coupled nonlinear oscillators. Phys Rev E Stat Nonlin Soft Matter Phys 2006;73: 065201 .

Zschocke S. Klinische Elektroenzephalographie. Berlin, Germany: Springer, 2002. 